\title{
From PID to State FeEdBack ATtitude STABILIZATION OF A QUADROTOR UAV
}

\author{
Fouad Yacef $^{1}$, Hana Boudjedir ${ }^{1}$, Hicham Khebbache $^{2}$, and Omar Bouhali ${ }^{1}$ \\ ${ }^{1}$ Automatic Laboratory of Jijel (LAJ), Automatic Control Department, Jijel University, \\ ALGERIA \\ yaceffouad@yahoo.fr, bouhali_omar@yahoo.fr, hana_boudjedir@yahoo.fr \\ ${ }^{2}$ Automatic Laboratory of Setif (LAS), Electrical Engineering Department, Setif \\ University, ALGERIA \\ khebbachehichameyahoo.fr
}

\begin{abstract}
The work proposed in this paper aim to design tow controllers for attitude stabilization of a Quadrotor UAV. The first is a classical PID controller; the second is a nonlinear state feedback controller which designed using multiple model approach. We start with the design of a Takagi-Sugeno (T-S) model for Quadrotor modelling, and then we use Linear Matrix Inequality (LMI), and PDC (Parallel Disturbance Compensation) technique to design a nonlinear state feedback controller with pole placement in a prespecified region of the operating space. The requirements of stability and pole-placement in LMI region are formulated based on the Lyapunov direct method. By recasting these constraints into LMIs, we formulate an LMI feasibility problem to calculate the controller gains. The both controllers are applied to a nonlinear Quadrotor system. Simulation results show that the proposed LMI-based design controller (State feedback) yields better transient performance than those of PID controller.
\end{abstract}

\section{KEYWORDS}

Linear Matrix Inequality (LMI), Parallel Disturbance Compensation (PDC), PID Controller, Pole Placement, Quadrotor, Takagi-Sugeno (T-S) model.

\section{INTRODUCTION}

Unmanned flying robots or vehicles (UAVs) are gaining increasing interest because of a wide area of possible applications. While the UAV market has first been driven by military applications and large expensive UAVs, recent results in miniaturization, Mechatronics and microelectronics also offer an enormous potential for small and inexpensive Micro-UAVs for commercial use. One type of aerial vehicle with a strong potential also for indoor flight is the rotorcraft and the special class of four-rotor aerial vehicles, also called Quadrotor. This helicopter is considered as one of the most popular UAV platform. This kind of helicopters is dynamically unstable, and therefore suitable control methods were used to make them stable, as back-stepping and sliding-mode techniques [1] [2].

A complex problem can be solved by many strategy; divide \& conquer strategy is one of them. The problem is divided into simpler parts, which are solved independently and together yields the solution to the whole problem. The same strategy can be used for modelling and control of nonlinear systems, where the non-linear plant is substituted by locally valid set of linear sub models 
[3]. The model should be simple enough so that it can be easy understood. The accurate model that characterizes important aspects of the system being controlled is a necessary prerequisite for design of a controller. The idea of approximation based on Multiple Model Approach (MMA) is not new. Since the publication Johansen and Foss, the multiple-models approach knew an unquestionable interest. The Multiple Model approach appears in the literature under many different names, including Takagi-Sugeno (T-S) model [4], local model networks or operating regime decomposition.

Many works have been carried out to investigate the stability analysis and the design of state feedback controller of Takagi-Sugeno systems. Using a quadratic Lyapunov function and PDC (Parallel Disturbance Compensation) technique, sufficient conditions for the stability and stabilisability have been established [5] [6]. The stability depends on the existence of a common positive definite matrix guarantying the stability of all local subsystems. The PDC control is a nonlinear state feedback controller. The gain of this controller can be expressed as the solution of a linear matrix inequality (LMIs) set [7].

In this work, we present a T-S model for Quadrotor modelling. A nonlinear state feedback controller is proposed, based on linear control theory, and PDC technique we formulate an LMI feasibility problem which considered as an optimization problem. A comparison study between PID and state feedback controller is made to proved performances of the proposed controller.

\section{QUADROTOR DYNAMICAL MODEL}

The Quadrotor is a Mechatronics system with four propellers in a cross configuration. While the front and the rear motor rotate clockwise, the left and the right motor rotate counter clockwise which nearly cancels gyroscopic effects and aerodynamic torques in trimmed flight. One additional advantage of the Quadrotor compared to a conventional helicopter is the simplified rotor mechanics. By varying the speed of the single motors, the lift force can be changed and vertical and/or lateral motion can be created. Pitch movement is generated by a difference between the speed of the front and the rear motor while roll movement results from differences between the speed of the left and right rotor, respectively.

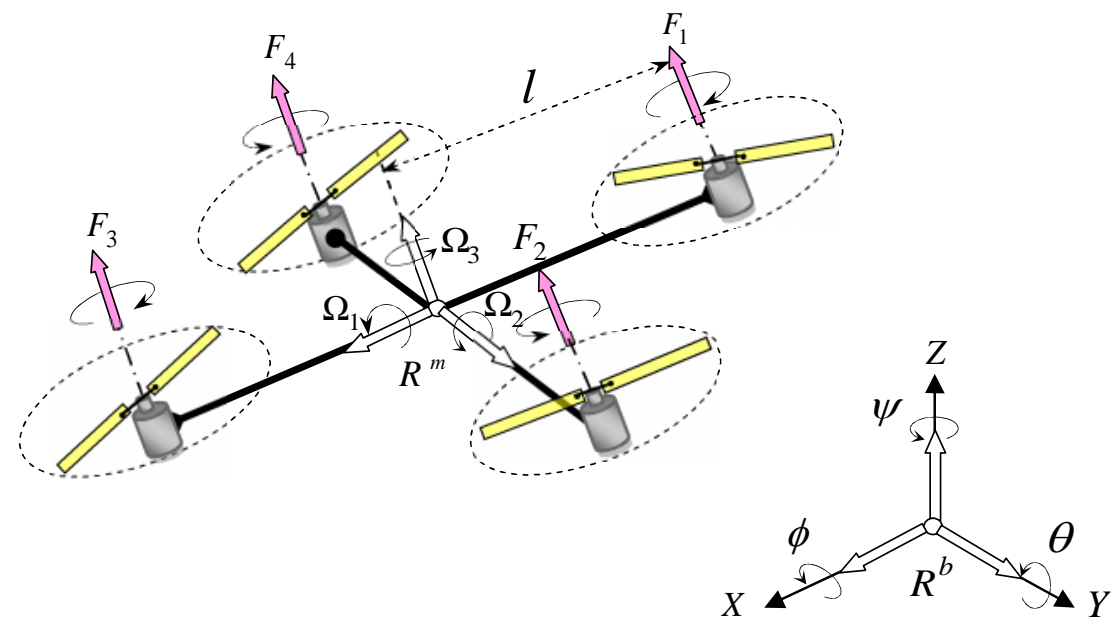

Figure 1. Quadrotor Architecture

The dynamics of the Quadrotor is described in the space by six degrees of freedom according to the fixed inertial frame related to the ground. This dynamics is related to the translational 
positions $(x, y, z)$ and the attitude described by the Euler angles $(\phi, \theta, \psi)$. These six coordinates are the absolute position of the centre of masse. The Euler angles are defined as follows:

- Roll angle $\phi:-\pi^{\prime} 2 \leq \phi \leq \pi^{\prime} 2$;

- Pitch angle $\theta:-\pi^{\prime} 2 \leq \theta \leq \pi^{\prime} 2$;

- Yaw angle $\psi:-\pi \leq \psi \leq \pi$.

The rotation transformation matrix $R$ from the inertial fixed frame $R^{b}$ to the body fixed frame $R^{m}$ is given by:

$$
R=\left[\begin{array}{ccc}
c \psi c \theta & s \phi s \theta c \psi-s \psi c \phi & c \phi s \theta c \psi+s \psi s \phi \\
s \psi c \theta & s \phi s \theta s \psi+c \psi c \theta & c \phi s \theta s \psi-s \phi c \psi \\
-s \theta & s \phi c \theta & c \phi c \theta
\end{array}\right]
$$

With $s($.$) and c($.$) represent \sin ($.$) and \cos ($.$) respectively.$

To derive the dynamic model of the Quadrotor, the Newton Euler formalism will be used on both translation and rotation motions. In this work we mainly focus our interest to the attitude dynamics and we consider the reduced dynamical model as follows [8]:

$$
\left\{\begin{array}{l}
\ddot{\phi}=\dot{\theta} \dot{\psi} \frac{\left(I_{y}-I_{z}\right)}{I_{x}}-\frac{I_{r}}{I_{x}} \Omega_{r} \dot{\theta}-\frac{K_{f a x}}{I_{x}} \dot{\phi}^{2}+\frac{1}{I_{x}} u_{1} \\
\ddot{\theta}=\dot{\phi} \dot{\psi} \frac{\left(I_{z}-I_{x}\right)}{I_{y}}+\frac{I_{r}}{I_{y}} \Omega_{r} \dot{\phi}-\frac{K_{f a y}}{I_{y}} \dot{\theta}^{2}+\frac{1}{I_{y}} u_{2} \\
\ddot{\psi}=\dot{\theta} \dot{\phi} \frac{\left(I_{x}-I_{y}\right)}{I_{z}}-\frac{K_{f a z}}{I_{z}} \dot{\psi}^{2}+\frac{1}{I_{z}} u_{3}
\end{array}\right.
$$

The inputs of the system are $u_{1}, u_{2}, u_{3}$ and $\Omega_{r}$ as a disturbance, obtaining:

$$
\left\{\begin{array}{l}
u_{1}=b l\left(\omega_{4}^{2}-\omega_{2}^{2}\right) \\
u_{2}=b l\left(\omega_{3}^{2}-\omega_{1}^{2}\right) \\
u_{3}=d\left(\omega_{1}^{2}-\omega_{2}^{2}+\omega_{3}^{2}-\omega_{4}^{2}\right) \\
\Omega_{r}=\omega_{1}-\omega_{2}+\omega_{3}-\omega_{4}
\end{array}\right.
$$

\section{QUADROTOR TAKAGI-SUGENO MODEL}

\subsection{Takagi-Sugeno model}

A T-S model is based on the interpolation between several LTI (linear time invariant) local models as follow:

$$
\dot{x}_{m}(t)=\sum_{i=1}^{r} \mu_{i}(\xi(t))\left(A_{i} x_{i}(t)+B_{i} u(t)\right)
$$


Where $r$ is the number of sub-models, $x_{m}(t) \in \mathbb{R}^{p}$ is the state vector, $u(t) \in \mathbb{R}^{h}$ is the input vector $A_{i} \in \mathbb{R}^{p \times p}, B_{i} \in \mathbb{R}^{p \times h}$, and $\xi(t) \in \mathbb{R}^{q}$ is the decision variable vector.

The variable $\xi(t)$ may represent measurable states and/or inputs and the form of this variable may leads to different class of systems: if $\xi(t)$ is known functions than the model (4) represents a nonlinear system and if there are unknown we consider that this leads to linear differential inclusion (LDI). This variable can also be a function of the measurable outputs of the system. The normalized activation function $\mu_{i}(\xi(t))$ in relation with the ith sub-model is such that:

$$
\left\{\begin{array}{l}
\sum_{i=1}^{r} \mu_{i}(\xi(t))=1 \\
0 \leq \mu_{i}(\xi(t)) \leq 1
\end{array}\right.
$$

According to the zone where evolves the system, this function indicates the more or less important contribution of the local model corresponding in the global model (T-S model).

The global output of T-S model is interpolated as follows:

$$
y_{m}(t)=\sum_{i=1}^{r} \mu_{i}(\xi(t))\left(C_{i} x_{i}(t)+D_{i} u(t)\right)
$$

Where $y_{m}(t) \in \mathbb{R}^{l}$ is the output vector and $C_{i} \in \mathbb{R}^{l \times p}, D_{i} \in \mathbb{R}^{l \times h}$. More detail about this type of representation can be found in [4].

\subsection{Quadrotor Takagi-Sugeno model}

The behaviour of a nonlinear system near an operating point $\left(x_{i}, u_{i}\right)$, can be described by a linear time-invariant system (LTI). Using Taylor series about $\left(x_{i}, u_{i}\right)$ and keeping only the linear terms yields:

$$
\dot{x}(t)=A_{i}\left(x(t)-x_{i}\right)+B_{i}\left(u(t)-u_{i}\right)+f\left(x_{i}, u_{i}\right)
$$

Which can written as

$$
\dot{x}(t)=A_{i} x(t)+B_{i} u(t)+d_{i}
$$

With:

$$
A_{i}=\left.\frac{\partial f(x, u)}{\partial x}\right|_{\substack{x=x_{i} \\ u=u_{i}}}, B_{i}=\left.\frac{\partial f(x, u)}{\partial u}\right|_{\substack{x=x_{i} \\ u=u_{i}}}, f(x, u)=\dot{x}(t), d_{i}=f\left(x_{i}, u_{i}\right)-A_{i} x_{i}-B_{i} u_{i}
$$

After calculation we obtained: 
International Journal of Information Technology, Control and Automation (IJITCA) Vol.2, No.3, July 2012

$$
A_{i}=\left[\begin{array}{cccccc}
0 & 1 & 0 & 0 & 0 & 0 \\
0 & a_{1} & 0 & a_{2} \dot{\psi}+a_{3} & 0 & a_{2} \dot{\theta} \\
0 & 0 & 0 & 1 & 0 & 0 \\
0 & a_{5}+a_{4} \dot{\psi} & 0 & a_{6} & 0 & a_{4} \dot{\phi} \\
0 & 0 & 0 & 0 & 0 & 1 \\
0 & a_{7} \dot{\theta} & 0 & a_{7} \dot{\phi} & 0 & a_{8}
\end{array}\right], \quad B_{i}=\left[\begin{array}{ccc}
0 & 0 & 0 \\
b_{1} & b_{2} & b_{3} \\
0 & 0 & 0 \\
b_{4} & b_{5} & b_{6} \\
0 & 0 & 0 \\
0 & 0 & b_{7}
\end{array}\right]
$$

Combined local affine models (8) using Gaussian activation function we describe the dynamic model of the Quadrotor by a T-S model:

$$
\left\{\begin{array}{l}
x_{m}(t)=\sum_{i=1}^{3} \mu_{i}(\xi(t))\left(A_{i} x_{m}(t)+B_{i} u(t)+d_{i}\right) \\
y_{m}(t)=C x_{m}(t)
\end{array}\right.
$$

With:

$$
\mu_{i}(\xi(t))=\frac{\omega_{i}(\xi(t))}{\sum_{j=1}^{3} \omega_{i}(\xi(t))}, \omega_{i}(\xi(t))=\prod_{j=1}^{3} \exp \left(-\frac{\left(\xi_{j}(t)-c_{i, j}\right)^{2}}{2 \sigma_{i, j}^{2}}\right)
$$

- The vector of decision variables $\xi(t)=\left[\begin{array}{lll}\dot{\phi} & \dot{\theta} & \dot{\psi}\end{array}\right]^{T}$

- The parameters of activations functions $\left(c_{i, j}, \sigma_{i, j}\right)$ are given as:

- The centres $c_{i, j}$ are defined according to the operation point.

- The Dispersions $\sigma_{i, j}$ are defined by optimization of a criterion, which represent the quadratic error between Takagi-Sugeno model outputs and nonlinear system outputs, using Particle Swarm Optimisation algorithm (PSO) [9].

-The operating points are chosen to cover maximum space of the operating space, with small number of local models. The attitude of Quadrotor (roll, pitch, and yaw) has a limited bound $\left(-\pi^{\prime} 2 \leq \phi \leq \pi^{\prime} 2,-\pi^{\prime} 2 \leq \theta \leq \pi^{\prime} 2,-\pi \leq \psi \leq \pi\right)$, for this reason we use three local models to cover this space. Linear local model are defined in this table as follow:

Table 1. Operation Points Parameters.

\begin{tabular}{|c|c|}
\hline $\mathbf{N}^{\circ} \mathbf{O . P}$ & Parameters \\
\hline 1 & $\dot{\phi}=\dot{\theta}=\dot{\psi}=-0.523 \mathrm{rad} / \mathrm{s}$ \\
\hline 2 & $\dot{\phi}=\dot{\theta}=\dot{\psi}=0 \mathrm{rad} / \mathrm{s}$ \\
\hline 3 & $\dot{\phi}=\dot{\theta}=\dot{\psi}=0.523 \mathrm{rad} / \mathrm{s}$ \\
\hline
\end{tabular}

To validate the synthesized T-S model a SBPA (input signal) is used, more detail in [10]. 


\section{Controller Design}

\subsection{PID controller}

A proportional-integral-derivative controller (PID controller) is a generic control loop feedback mechanism (controller) widely used in industrial control systems, a PID is the most commonly used feedback controller. A PID controller calculates an "error" value as the difference between a measured process variable and a desired trajectory. The controller attempts to minimize the error by adjusting the process control inputs.

The PID controller calculation (algorithm) involves three separate constant parameters, and is accordingly sometimes called three-term control: the proportional, the integral and derivative values, denoted P, I, and D. In the absence of knowledge of the underlying process, a PID controller has historically been considered to be the best controller.

The control law can be given as:

$$
U_{P I D}=K_{p} e(t)+K_{i} \int_{0}^{t} e(\tau) d \tau+K_{d} \frac{d e(t)}{d t}
$$

Where: $K_{p}$ : Proportional gain; $K_{i}:$ Integral gain; $K_{d}$ : Derivative gain; $e$ : Error.

\subsection{State feedback controller}

The concept of PDC, following the terminology [5], is utilized to design state-feedback controller on the basis of the T-S model (10). Linear control theory can be used to design the control law, because T-S model is described by linear state equations. The controller law is a convex linear combination of the local controller associated with the corresponding local sub-model. It can present as:

$$
U(t)=-\sum_{i=1}^{r} \mu_{i}(\xi(t)) u_{i}(t)=-\sum_{i=1}^{r} \mu_{i}(\xi(t)) K_{i} x(t)
$$

With: $K_{i}$ is $r$ vector of feedback gains.

It should be noted that the designed controller shares the same models sets with T-S models, and resulting controller (12) is nonlinear in general since the coefficient of the controller depends nonlinearly on the system input and output via the weighting functions. Substituting (12) into (10), the closed-loop T-S model can be represented by:

$$
\dot{x}(t)=\sum_{i=1}^{r} \sum_{j=1}^{r} \mu_{i}(\xi(t)) \mu_{j}(\xi(t))\left(A_{i}-B_{i} K_{j}\right) x(t)
$$

The constant $d_{i}$ was neglected in this formulate, because the control law can compensate the effect of this bias term.

\subsubsection{Stabilisation using PDC}

A sufficient quadratic stability condition derived by Tanaka and Sugeno [11] for ensuring stability of (13) is given as follows: 
Theorem 1: The closed-loop T-S model (13) is quadratic-ally stable for some stable feedback $K_{i}$ (via PDC scheme) if there exists a common positive definite matrix $P$ such that:

$$
\begin{gathered}
G_{i i}^{T} P+P G_{i i}<0 \quad \forall i \in I_{r} \\
\left(\frac{G_{i j}+G_{j i}}{2}\right)^{T} P+P\left(\frac{G_{i j}+G_{j i}}{2}\right)<0, \quad \forall(i, j) \in I_{r}^{2}, i<j
\end{gathered}
$$

With: $G_{i i}=A_{i}-B_{i} K_{j}, \mu_{i}(\xi(t)) \mu_{j}(\xi(t)) \neq 0$.

Which is an LMI in $P$ when $K_{i}$ are predetermined. However, our objective is to design the gain matrix $K_{i}$ such that conditions (14) are satisfied. That is, $K_{i}$ are not pre-determined matrices any longer, but matrix variables. This is the quadratic stability problem and can be recast as an LMI feasibility problem. With linear fractional transformation $X=P^{-1}$ and $N_{i}=K_{i} X$, we may rewrite (14) as an LMI problem in $N_{i}, X$ and $S_{i j}$ [12]:

$$
\begin{gathered}
X>0 \\
X A_{i}{ }^{T}+A_{i} X-N_{i}^{T} B_{i}^{T}-B_{i} N_{i}+S_{i i}<0, \quad \forall i \in I_{r} \\
X A_{i}{ }^{T}+A_{i} X+X A_{j}{ }^{T}+A_{j} X-N_{j}^{T} B_{i}^{T}-B_{i} N_{j} \\
-N_{i}^{T} B_{j}^{T}-B_{j} N_{i}+2 S_{i j} \leq 0, \quad \forall(i, j) \in I_{r}^{2}, i<j \\
\left(\begin{array}{ccc}
S_{11} & \cdots & S_{1 n} \\
\vdots & \ddots & \vdots \\
S_{1 n} & \cdots & S_{n n}
\end{array}\right)>0
\end{gathered}
$$

With: $S_{i j}=X Q_{i j} X, \forall i \in\{1, \ldots, r\}, Q_{i j}$ are symmetric matrix.

\subsubsection{LMI formulation for Pole placement}

In order to achieve some desired transient performance, a pole placement should be considered. For many problems, exact pole assignment may not be necessary; it suffices to locate the pole of the closed loop system in a sub-region of the complex left half plane. This section discusses a pole assignment in LMI regions. For this purpose, we introduce the following LMI-based representation of stability regions [13] [14].

Definition: A subset $D$ of the complex plane is called an LMI region if there exist a symmetric matrix $\alpha=\left(\alpha_{i j}\right) \in \mathbb{R}^{p . p}$ and a matrix $\beta=\left(\beta_{i j}\right) \in \mathbb{R}^{p . p}$ such that:

$$
D=\left\{z \in \sqcup: f_{D}(z)<0\right\}
$$

Where: $f_{D}(z)=\left(\alpha_{i j}+\beta_{i j} z+\beta_{i j} \bar{z}\right), \forall i, j \epsilon\{1, \ldots, p\}$

Theorem 2: A matrix $A$ is D-stable if and only if there exists a symmetric positive definite matrix $X$ such that:

$$
M_{D}(X, A)<0
$$


Where: $M_{D}(X, A)=\alpha \otimes X+\beta \otimes(A X)+\beta^{T} \otimes(A X)^{T}$

For example, a circle region $D$ centred at $(-q, 0)$ with radius $\rho>0$ can be obtained by taking the matrices and as follows:

$$
\alpha=\left(\begin{array}{cc}
-\rho & q \\
q & -\rho
\end{array}\right) \text {, and } \beta=\left(\begin{array}{ll}
0 & 1 \\
0 & 0
\end{array}\right)
$$

What makes it possible to obtain the expression of the characteristic function:

$$
f_{D}(z)=\left(\begin{array}{cc}
-\rho & z^{*}+q \\
z+q & -\rho
\end{array}\right)
$$

As it is shown in figure 2, this region which include conic region, allows fixing a lower bound on both the exponential decay rate: $\rho-q$ and the damping ratio: $\zeta_{\min }=\sqrt{1-\left(\rho^{2 \top} q^{2}\right)}(\rho<q)$ of the closed-loop response, and thus is very common in practical control design.

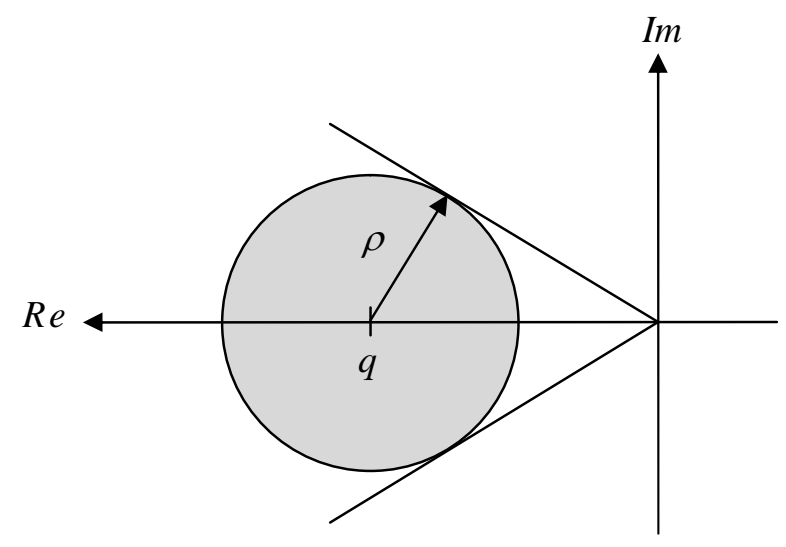

Figure 2. Circular region $(D)$ for pole location

Since the prescribed LMI region (18) will be added as supplementary constraints to these of the theorem 1 , it should be noted that it only suffices to locate the poles of the dominant term in the prescribe LMI regions, i.e. the case of $i=j$. It follows that the closed loop T-S model (13) is Dstable if there exists a symmetric matrix $X$ such that [15]:

$$
\left(\begin{array}{cc}
-\rho X & q X+X\left(A_{i}+B_{i} K_{j}\right)^{T} \\
q X+\left(A_{i}+B_{i} K_{j}\right) X & -\rho X
\end{array}\right)
$$

With the same change of variables $N_{i}=K_{i} X$ leads to the following LMI formulation:

$$
\left(\begin{array}{cc}
-\rho X & q X+X A_{i}^{T}+B_{i}^{T} N_{i}^{T} \\
q X+X A+B_{i} N_{i} & -\rho X
\end{array}\right), i=j
$$

By combining Theorems 1 and 2 leads to the following LMI formulation of two objectives statefeedback synthesis problem, such that the resulting controller meets both the global stability and 
the desired transient performance simultaneously. The closed loop T-S model (13) is stabilizable in the specified region $D$ if there exists a symmetric matrix $X$ such that [13]:

$$
\begin{gathered}
X>0 \\
X A_{i}{ }^{T}+A_{i} X-N_{i}^{T} B_{i}^{T}-B_{i} N_{i}+S_{i i}<0, \quad \forall i \in I_{r} \\
X A_{i}{ }^{T}+A_{i} X+X A_{j}{ }^{T}+A_{j} X-N_{j}^{T} B_{i}^{T}-B_{i} N_{j} \\
-N_{i}^{T} B_{j}^{T}-B_{j} N_{i}+2 S_{i j} \leq 0, \quad \forall(i, j) \in I_{r}^{2}, i<j \\
\left(\begin{array}{cc}
-\rho X & q X+X A_{i}^{T}+N_{i}^{T} B_{i}^{T} \\
q X+A_{i} X+B_{i} N_{i} & -\rho X
\end{array}\right)<0, \quad \forall i \in I_{r} \\
\left(\begin{array}{ccc}
S_{11} & \cdots & S_{1 n} \\
\vdots & \ddots & \vdots \\
S_{1 n} & \cdots & S_{n n}
\end{array}\right)>0
\end{gathered}
$$

With: $S_{i j}=X Q_{i j} X, K_{i}=N_{i} X, \forall i \in\{1, \ldots, r\}$

\subsubsection{State feedback gains calculation}

Using Theorem 1 and 2; can design a nonlinear state feedback controller that guarantees global stability while provides desired transient behaviour by constraint the closed-loop poles in $D$. The stability region $D$ is a circle of centre $(q, 0)$ and radius $\rho$ and the LMI synthesis is performed for a set of values $(q, \rho)=(4,1)$.

Then the LMI region has the following characteristic function:

$$
f_{D}(z)=\left(\begin{array}{cc}
-1 & z^{*}+4 \\
z+4 & -1
\end{array}\right)
$$

This circle region puts a lower bound on both exponential decay rate $q-\rho=3 \mathrm{rad}^{\prime} \mathrm{s}$ and damping ratio $\zeta=\sqrt{1-\left(\rho^{2 \iota^{\prime}} q^{2}\right)}=0.97$ of the closed-loop response. By solving LMI feasibility problem (21), we can obtain a positive symmetric matrix $X$ (by interior-point method in Matlab LMI-toolbox), and stat feedback Matrix $K_{i}$ as:

$$
\begin{aligned}
& K_{1}=\left[\begin{array}{cccccc}
0.05614 & 0.02879 & 0 & 0.2004 \times 10^{-2} & -0.01528 & -0.5897 \times 10^{-2} \\
0 & -0.00200 & 0.05615 & 0.02879 & 0.01528 & 0.5897 \times 10^{-2} \\
0 & 0.5236 \times 10^{-6} & 0 & 0.5236 \times 10^{-6} & 0.11229 & 0.05806
\end{array}\right] \\
& K_{2}=\left[\begin{array}{cccccc}
0.05614 & 0.02879 & 0 & 0 & 0 & 0 \\
0 & 0 & 0.05615 & 0.02879 & 0 & 0 \\
0 & 0 & 0 & 0 & 0.11229 & 0.05806
\end{array}\right] \\
& K_{3}=\left[\begin{array}{cccccc}
0.05614 & 0.02879 & 0 & -0.2004 \times 10^{-2} & 0.01528 & 0.5897 \times 10^{-2} \\
0 & 0.00200 & 0.05615 & 0.02879 & -0.01528 & -0.5897 \times 10^{-2} \\
0 & -0.5236 \times 10^{-6} & 0 & -0.5236 \times 10^{-6} & 0.11229 & 0.05806
\end{array}\right]
\end{aligned}
$$


International Journal of Information Technology, Control and Automation (IJITCA) Vol.2, No.3, July 2012

\subsection{Simulation results}

The controller described above was simulated for the nonlinear Quadrotor system. Simulations are made for initial values equal to $(\phi, \theta, \psi)=(30,-20,30) \mathrm{deg}$ for stabilization, and equal to zeros for tracking. The values of the model parameters used for simulations are the following:

$$
\begin{aligned}
& m=0.486 \mathrm{~kg}, l=0.225 \mathrm{~m}, g=9.81 \mathrm{~m} / \mathrm{s}^{2}, d=3.23 \times 10^{-7} \mathrm{~N} \cdot \mathrm{m} /(\mathrm{rad} . \mathrm{s}), b=2.98 \times \\
& 10^{-5} \mathrm{~N}^{\prime}(\mathrm{rad} . \mathrm{s}), I_{x}=I_{y}=3.82 \times 10^{-3} \mathrm{~kg} \cdot \mathrm{m}^{2}, I_{z}=7.65 \times 10^{-3} \mathrm{~kg} \cdot \mathrm{m}^{2}, K_{\text {fax }}=K_{\text {fay }}= \\
& 5.567 \times 10^{-4}, K_{\text {faz }}=6.354 \times 10^{-4} .
\end{aligned}
$$
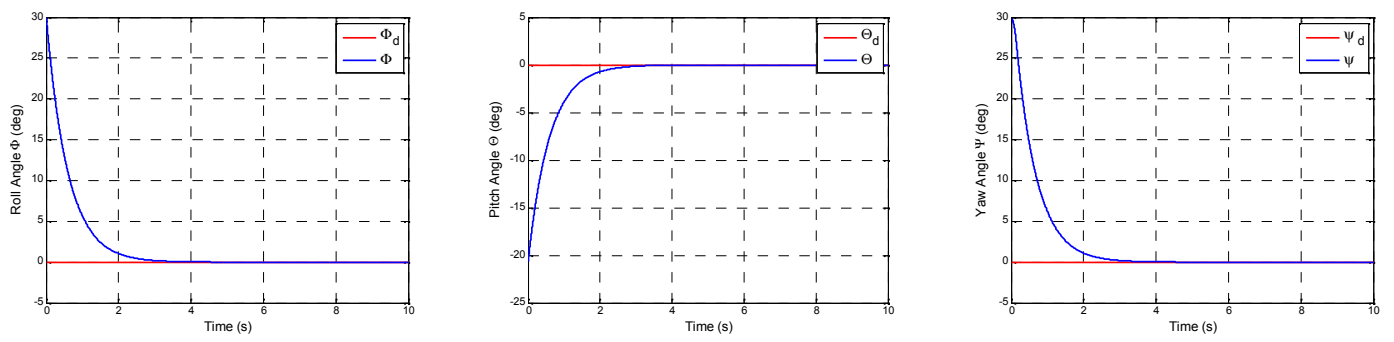

Figure 3. Quadrotor Attitude $(\phi, \theta, \psi)$ for PID controller
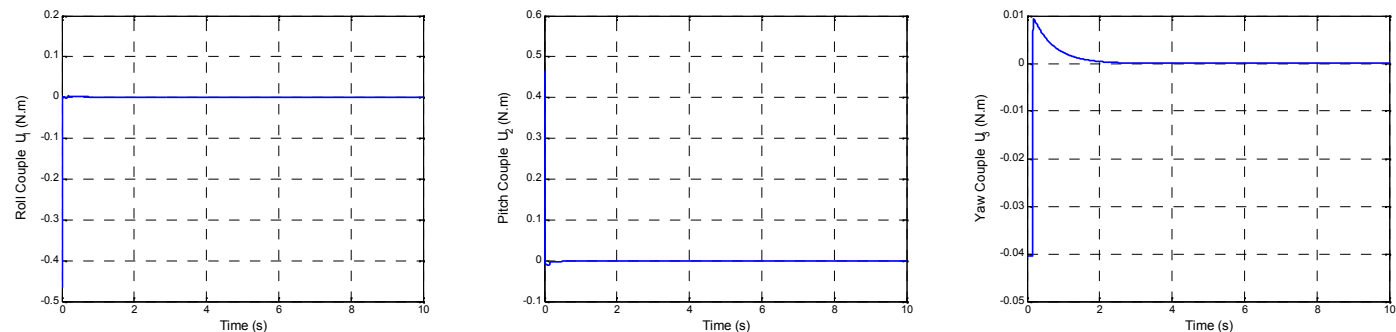

Figure 4. Quadrotor control inputs $\left(u_{1}, u_{2}, u_{3}\right)$ for PID controller

The results of PID controller and the corresponding result of state feedback controller are shown in Figure 3 and Figure 4 respectively which indicates the output of Quadrotor nonlinear system (Quadrotor attitude, Roll, Pitch, and Yaw), and the corresponding control inputs in figure 5, figure 6 .

From these results, it can be noticed that state the feedback controller provides better transient performance (stabilization time, overtaking...) than those of PID controller, while both controller give stable response regardless of any initial displacement. The control inputs are smoother and realizable.
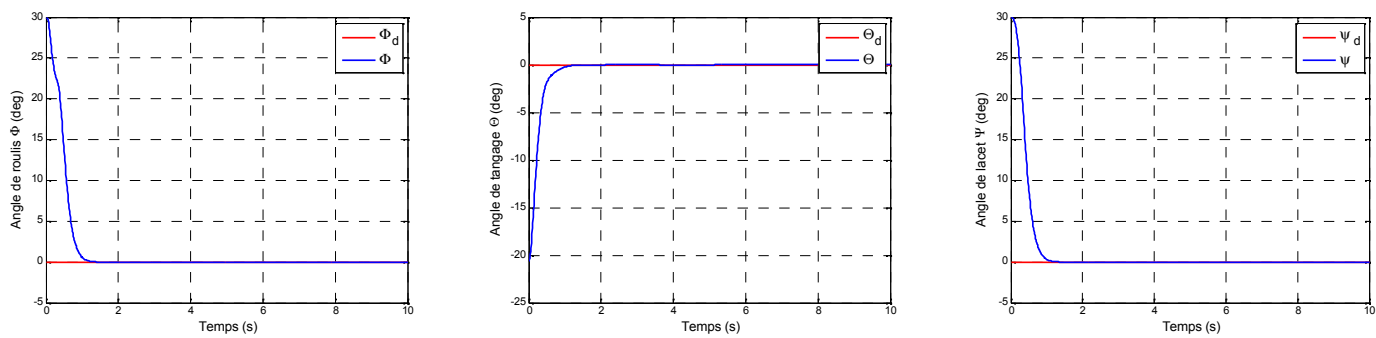

Figure 5. Quadrotor Attitude $(\phi, \theta, \psi)$ for state feedback controller 

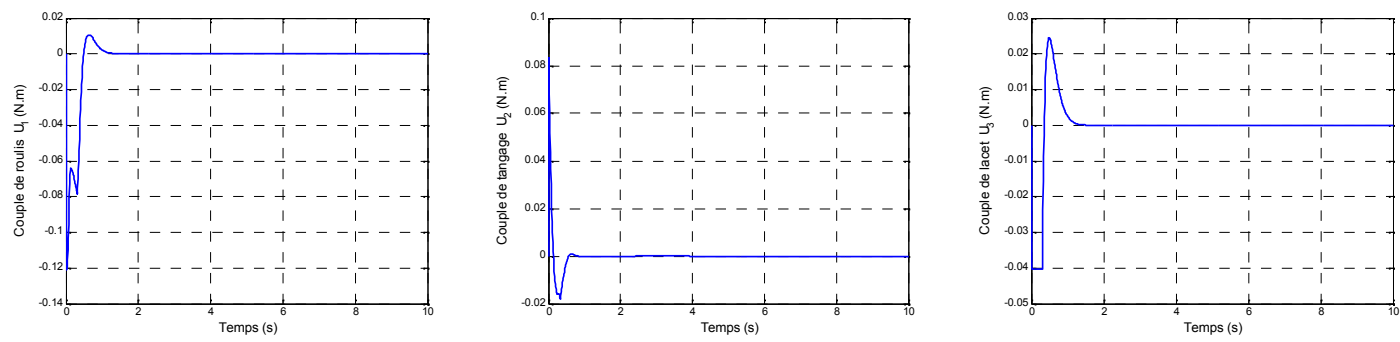

Figure 6. Quadrotor control inputs $\left(u_{1}, u_{2}, u_{3}\right)$ for state feedback controller

Figure 7 represent Quadrotor attitude tracking (Roll, Pitch, and Yaw), for a sinusoidal trajectory, it can be noticed that the designed controller provides good tracking performances. Figure 8 represent inputs control system.
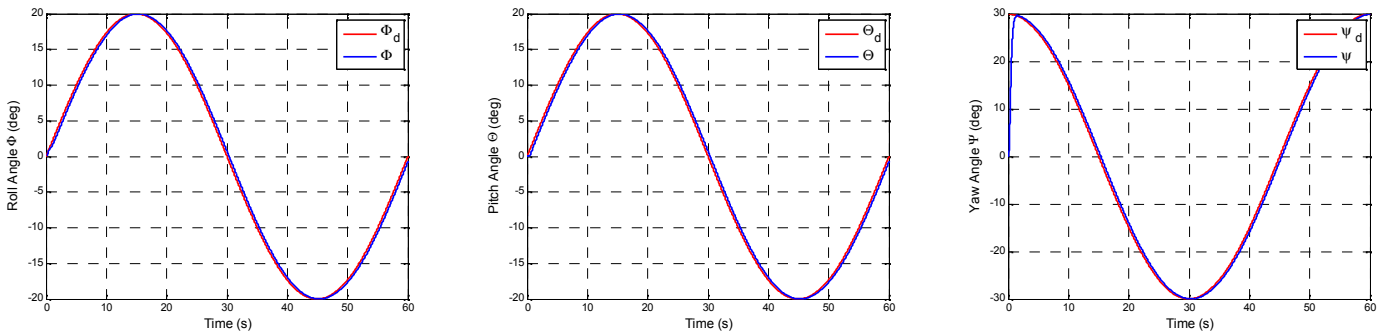

Figure 7. Quadrotor Attitude $(\phi, \theta, \psi)$ for state feedback controller
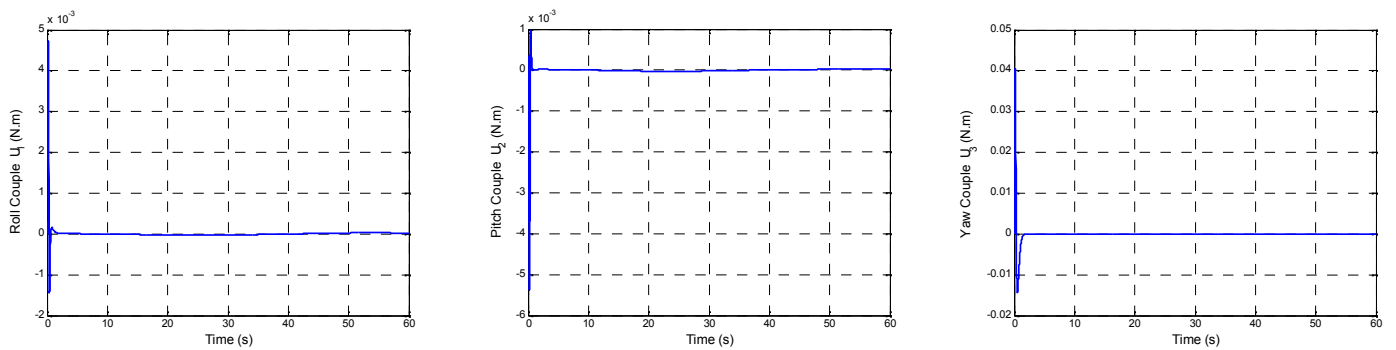

Figure 8. Quadrotor control inputs $\left(u_{1}, u_{2}, u_{3}\right)$ for state feedback controller

\section{CONCLUSIONS}

In this paper the stabilization of Quadrotor attitude represented by a T-S model is considered. For that we use tow controllers: a classical PID controller, and a state feedback controller. For de design of the state feedback controller a systematic design methodology is used with guaranteed stability and pre-specified transient performance. The framework is based on Parallel Distributed Compensation (PDC) technique and pole placement in LMI region. Simulation results showed that the state feedback controller provides not only maximized stability but also better transient performance than PID controller.

\section{APPENDIX}

Parameters of $A_{i}$ and $B_{i}$ matrix 
International Journal of Information Technology, Control and Automation (IJITCA) Vol.2, No.3, July 2012

$$
\left\{\begin{array}{l}
a_{1}=-\frac{K_{f a x}}{I_{x}} \\
a_{2}=\frac{\left(I_{y}-I_{z}\right)}{I_{x}} \\
a_{3}=-\frac{J_{r}}{I_{x}} \Omega_{r} \\
a_{4}=\frac{\left(I_{z}-I_{x}\right)}{I_{y}}
\end{array},\left\{\begin{array}{l}
a_{5}=\frac{J_{r}}{I_{y}} \Omega_{r} \\
a_{6}=\frac{K_{f a y}}{I_{y}} \\
a_{7}=\frac{\left(I_{x}-I_{y}\right)}{I_{z}} \\
a_{8}=-\frac{K_{f(c)}}{I_{z}}
\end{array},\left\{\begin{array}{l}
b_{1}=-\frac{J_{r}}{I_{x}} \dot{\theta} C_{1}+\frac{1}{I_{x}} \\
b_{2}=-\frac{J_{r}}{I_{x}} \dot{\theta} C_{2} \\
b_{3}=\frac{J_{r}}{I_{x}} \dot{\theta} C_{3} \\
b_{4}=\frac{J_{r}}{I_{y}} \dot{\phi} C_{1}
\end{array}, \quad\left\{\begin{array}{l}
b_{5}=-\frac{J_{r}}{I_{y}} \dot{\phi} C_{2}+\frac{1}{I_{y}} \\
b_{y}=-J_{r} \\
b_{7}=\frac{1}{I_{z}}
\end{array}\right.\right.\right.\right.
$$

\section{REFERENCES}

[1] T. Madani \& A. Benallegue, (2006) "Backstepping Sliding Mode Control Applied to a Miniature Quadrotor Flying Robot", IEEE Conference on Industrial Electronics, pp. 700-705.

[2] Y. Yu, J. Changhong, \& W. Haiwei, (2010) "Backstepping control of each channel for a Quadrotor aerial robot", International Conference on Computer, Macaronis, Control and Electronic Engineering (CMCE), pp. 403-407.

[3] J. Novák. (2007) "linear system identification and control using local model networks". A thesis submitted in fulfilment of the requirements for the $\mathrm{PhD}$ degree, Faculty of Applied Informatics, Tomas Bata University, Zlín, Czech.

[4] T. Takagi \& M. Sugeno, (1985) "Fuzzy identification of systems and its applications to model and control", IEEE Transactions on Systems, Man, and Cybernetics, vol. 15, pp. 116-132.

[5] H. O. Wang, K. Tanaka, \& M. Griffin, (1996) "An approache to fuzzy controle of non linear systems : stability and design issues," IEEE Transaction on fuzzy system, vol. 4, pp. 14-23.

[6] K. Tanaka, . T. Ikeda, \& Y. Y. He, (1998) "Fuzzy regulators and fuzzy observers : relaxed stability conditions and LMI-based design," IEEE Transaction on fuzzy system, vol. 6, pp. 250-256.

[7] S. Boyd, L. El Ghaoui, E. Feron, \& V. Balakrishnan (1994) "Linear Matrix Inequalities in System and Control Theory," SIAM, Philadelphia, USA.

[8] H .Bouadi, S. S. Cunha, A. Drouin, \& F. M. Camino, (2011) "Adaptive Sliding Mode Control for Quadrotor Attitude Stabilization and Altitude Tracking", IEEE International Symposium on Computational Intelligence and Informatics, pp. 449-455.S.

[9] F. Yacef \& F. Boudjema, (2011) "Local Model Network for non linear modelling and control of an UAV Quadrotor", International Conference on Automatic and Mechatronics (CIAM), November 2224, Oran, Algeria, pp. 247-252.

[10] F. Yacef, O. Bouhali, H. Khebbache \& F. Boudjema, (2012) "Takagi-Sugeno Model for Quadrotor Modelling and Control Using Nonlinear State Feedback Controller", International Journal of Control Theory and Computer Modelling (IJCTCM), May 2012. [Accepted]

[11] K. Tanaka \& M. Sugeno, (1992) "Stability analysis and design of fuzzy control systems", Fuzzy Set and Systems, vol. 45, pp. 135-156.

[12] M. Chadli, (2002) "Stability and control of multiple models: LMI approach", INPL thesis (in French), France. mchadli.voila.net/THESECHADLI.pdf.

[13] S. K. Hong \& Y. Nam, (2003) "Stable fuzzy control system design with pole-placement constraint: an LMI approach", Computers in Industry, vol. 51, pp. 1-11.

[14] M. Chadli, D. Maquin, J. Ragot, (2002) "Static Output Feedback for Takagi-Sugeno Systems: an LMI Approach", Proceedings of the 10th Mediterranean Conference on Control and Automation, July 912, Lisbon, Portugal.

[15] M. Chilali \& P. Gahinet, (1996) "Hळ Design with pole placement constraints: an LMI approach", IEEE Transaction on Automatic Control, vol. 41, pp. 358-367. 


\section{Authors}

Fouad Yacef is currently a Ph.D. student at the Automatic Control Department of Jijel University, Algeria. He received the Engineer degree in Automatic Control from Jijel University, Algeria in 2009, and Magister degree in Automatic Control from the Military Polytechnic School of Algeria (EMP), in November 2011. Since December 2011, he has been a researcher in Automatic Laboratory of Jijel (LAJ). His research interests include Aerial robotics, linear and nonlinear control, analysis and design of intelligent control and renewable energy systems.

Hana Boudjedir received the Engineer degree and Magister degree in Automatic Control from Jijel University, Algeria in 2008, and 2010, respectively. She is currently a Ph.D. student at the Automatic Control Department of Jijel University, Algeria. Since December 2011, she has been a researcher in Automatic Laboratory of Jijel (LAJ). Her research interests include aerial robotics, nonlinear control systems, analysis and design of intelligent control systems.

Hicham Khebbache is Graduate student (Magister) of Automatic Control at the Electrical Engineering Department of Setif University, Algeria. He received the Engineer degree in Automatic Control from Jijel University, Algeria in 2009. Now he is with Automatic Laboratory of Setif (LAS). His research interests include Aerial robotics, Linear and Nonlinear control, Robust control, Fault tolerant control (FTC), Diagnosis, Fault detection and isolation (FDI).

Omar Bouhali He received the engineer degree and the master degree in automatic from national polytechnic school of Algeria, in 1996 and 1998 respectively. He received the Ph. D. degree from the Central School of Lille, France and National Polytechnic School of Algeria in 2007. Since 1998, he has been a lecturer in the automatic department of Jijel University, Algeria. And he is a researcher in Automatic Laboratory of Jijel (LAJ). He is working in the development of renewable energy based power systems with inertial storage using multilevel converters.
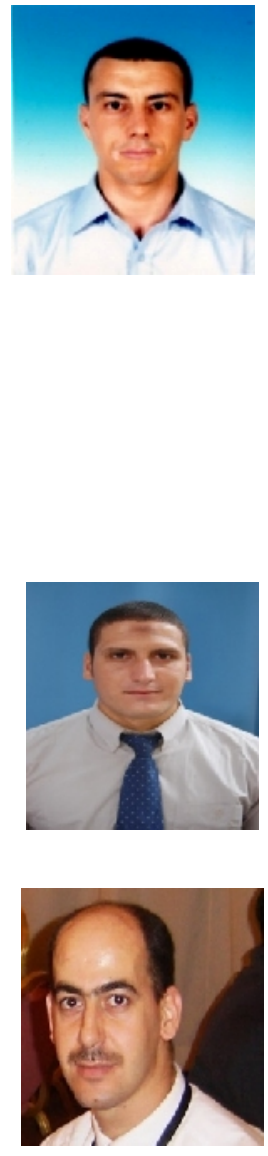